\title{
Caspase-3-Dependent and -Independent Apoptosis in Focal Brain Ischemia
}

\author{
Vladimir V. Didenko, Hop Ngo, Candace L. Minchew, Denise J. Boudreaux, Marsha A. Widmayer, \\ and David S. Baskin
}

Department of Neurosurgery and Surgical Service, Baylor College of Medicine and VAMC, Houston, Texas, USA

Accepted June 18, 2002

\begin{abstract}
Background: Although extensive caspase-3 activation has been demonstrated in experimental brain ischemia produced in neonatal rat, the role this caspase plays in the focal ischemia of adult brain is not clear, as the levels of caspase- 3 in adult rat brain are extremely low. This raises the question whether caspase- 3 synthesis and activation are essential for execution of the apoptotic program and DNA fragmentation in permanent brain ischemia, a condition that impairs cellular protein synthesis.

Materials and Methods: Rat middle cerebral artery was permanently occluded and histochemical detection of procaspase-3, active caspase-3 and DFF40/CAD and apoptotic morphology analysis were performed at 6, 24, 48, and 72 hours after occlusion.

Results: Necrosis and two types of programmed cell death (PCD) are identified in this study of permanent focal brain ischemia. The first type of PCD is represented by active caspase- 3 and DFF40/CAD-positive cells. The
\end{abstract}

second type of PCD is represented by caspase- 3 and DFF40/CAD negative cells, which display morphological signs of apoptosis-like PCD: namely, nuclear chromatin condensation in lump masses and apoptotic body formation. The cells of the first type have a maximum number noted after 24 hours of ischemia. The cells of the second type are primarily seen after 48 and 72 hours of ischemia. Necrotic cells, which are also detected in the stroke, are caspase-3 negative, and have swollen nuclei, without chromatin condensation and apoptotic body formation.

Conclusions: Our results indicate that in permanent brain ischemia in adult rats, PCD processes occur differently in various parts of ischemic zone. In conditions of severe energy depletion, the reactions of cellular disassembly and packaging into apoptotic bodies are accomplished without either caspase-3 expression or the activation of caspase-3dependent deoxyribonuclease.

\section{Introduction}

Apoptosis is an orderly process of cellular suicide. It is usually initiated by the activation of caspases, and produces DNA fragmentation and cell disassembly. Though the sequence of events in the caspase cascade may differ in various tissues, caspase- 3 is thought to be tightly linked to the final events in the execution cell death program via specific and extensive apoptotic DNA fragmentation(1). Caspase-3 initiates apoptotic DNA fragmentation by triggering its target executioner nuclease-a recently discovered caspase-activated deoxyribonuclease (CAD, or DFF 40) $(2,3)$. DFF 40 is kept inactive by the binding of an inhibitor (ICAD, or DFF 45). Activation of the nuclease occurs when its inhibitor, ICAD, is cleaved by caspase- $3(4,5)$.

The ability of caspase- 3 inhibitors to prevent apoptotic DNA fragmentation and subsequent cell death (6) has led to interest in the development of drugs aimed at controlling the apoptotic process via caspase- 3 inhibition $(7,8)$.

Address correspondence and reprint requests to: David S. Baskin, Department of Neurosurgery, 6560 Fannin, Suite 944, Houston, TX, 77030. Phone: 713-798-4696; fax: 713-798-3227;

e-mail: dbaskin@tmh.tmc.edu.
Ischemic brain represents one of the major targets of such therapies. Indeed, extensive caspase-3 activation was demonstrated to occur throughout the ischemic zone in experimental brain ischemia produced in neonatal rat, 7 days postpartum (9). However, the role this caspase plays in the focal ischemia of adult brain is not clear, as the levels of caspase- 3 diminish markedly 2 weeks after birth, and in adult rat brain are extremely low $(10,11)$. This raises the question of how necessary caspase- 3 synthesis and activation are for execution of the apoptotic program and DNA fragmentation in conditions of permanent brain ischemia, which impairs cellular protein synthesis.

In our experiments, rat middle cerebral artery was permanently occluded and histochemical detections of procaspase-3, active caspase-3 and DFF40/CAD and apoptotic morphology analysis were performed at 6 , 24, 48, and 72 hours after occlusion.

We demonstrate that necrotic and two types of apoptotic cells can be detected in the ischemic zone. Many cells located in the ischemic zone are caspase-3 and DFF40 positive. However, there are also many cells in the ischemic zone that are either morphologically necrotic or display a morphology different from classical apoptosis but characteristic for apoptosislike PCD (12). They exhibit nuclear fragmentation 
and formation of apoptotic bodies, but their chromatin condensation is less compact compared to the classical apoptosis and is analogous to lumpy chromatin condensation recently demonstrated in caspase-inhibited apoptotic neurons (13). Neither caspase-3 nor DFF40 are present in these cells.

This suggests a caspase-3- and DFF40-independent mechanism of execution of PCD with apoptoticlike cell dismantling under conditions of persistent energy depletion. Synthesis and activation of caspase-3 and subsequent activation of DFF40 in these conditions are impaired, whereas apoptotictype cell disassembly with packaging of cellular contents into apoptotic bodies can still occur and proceed in the cells in the core ischemic areas.

\section{Materials and Methods}

The animal care and use portion of the following protocol was reviewed and approved by the Institutional Animal Care and Use Committee of the VA Medical Center and the Animal Protocol Review Committee of Baylor College of Medicine. All procedures were carried out with strict adherence to the protocol and the National Institutes of Health animal research guidelines.

\section{Focal Brain Ischemia}

All surgical techniques were performed under 2\% isoflurane gas anesthesia. Occlusion of the right middle cerebral artery (MCA) was performed in eight, male, Long Evans rats through an incision made midway between the right eye and ear. Using the operating microscope, the right MCA was exposed transcranially via a subtemporal craniotomy without damage to the zygomatic bone. $10-12 \mathrm{~mm}$ of the MCA, beginning ventral to the olfactory tract, was permanently occluded using bipolar coagulation.

$6,24,48$, and 72 hours following occlusion, the animals were transcranially perfused with paraformaldehyde and the brains were removed and postfixed overnight. A $2 \mathrm{~mm}$ thick coronal block was cut from the center of the MCA territory, beginning $\sim 0.5 \mathrm{~mm}$ anterior to the optic chiasm and ending $\sim 0.5 \mathrm{~mm}$ posterior to the optic chiasm. The tissue was embedded in paraffin and cut in $6 \mu \mathrm{m}$ thick sections. Sections used in the current study were taken from the middle area of the tissue block. Each staining condition was repeated in 4 separate sections from each of the 8 animals.

\section{Immunohistochemistry and TUNEL Labeling}

Following paraformaldehyde perfusion and postfixation, a $2 \mathrm{~mm}$ thick coronal block was cut from the center of the MCA territory, beginning $0.5 \mathrm{~mm}$ anterior to the optic chiasm and ending $0.5 \mathrm{~mm}$ posterior to the optic chiasm. The tissue was embedded in paraffin and cut in $6 \mu \mathrm{m}$ thick sections. Sections used in the current study were taken from the middle area of the tissue block. The boundaries of the ischemic zone at every time point were visualized using cresyl violet staining.

For staining, brain sections were deparaffinized with xylene, rehydrated in graded alcohol concentrations, and washed in water. Microwave antigen retrieval was performed in $0.01 \mathrm{M}$ sodium citrate, pH 6.0 for $15 \mathrm{~min}$. Cleaved caspase-3 (17 kDa) polyclonal antibody (1:100) (cat\#96615 New England Biolabs), caspase- 3 p20 and caspase- 3 p1l polyclonal antibodies (cat\# sc-1225 and sc1224, Santa Cruz Biotechnology, Inc.) (both at 1:50 dilution), and DFF 40 polyclonal antibody (cat\# scl443) (1:100 dilution) were used in the experiments. For cleaved caspase-3 and DFF 40, the signal was visualized using biotinylated goat-antirabbit antibody (1:500) and TSA ${ }^{\mathrm{TM}}$-direct kit (NEN) and for p20 and pl1 by using biotinylated donkey anti-goat antibody (1:50) and streptavidin-FITC.

For the TUNEL labeling, we used the ApoTaq kit (Intergen) as recommended by the manufacturer, with subsequent counterstaining with DAPI ( $1 \mu \mathrm{g} /$ $\mathrm{ml})$ to visualize nuclear morphology and apoptotic bodies.

\section{Electron Microscopy}

Small pieces of tissues cut from the paraffin blocks were placed in three changes of $100 \%$ xylene and then passed through a descending series of ethanols $(100 \%, 95,70,50$, water $)$ and placed in PBS. The tissue was then fixed in $2.5 \%$ glutaraldehyde in $0.1 \mathrm{M}$ cacodylate buffer, washed in $0.1 \mathrm{M}$ cacodylate buffer and postfixed in $2 \%$ osmium tetroxide in the same buffer. It was dehydrated in an ascending series of ethanols passed through 3 changes of propylene oxide and then embedded in epon (EMBED 812, Electron Microscopy Sciences). Ultrathin sections $(80 \mathrm{~nm})$ were cut with a diamond knife and RMC ultramicrotome. The sections were stained with lead citrate and alcoholic uranyl acetate. They were viewed and recorded on a Hitachi H7000 TEM.

\section{Results}

Using immunohistochemical staining for active caspase-3, we detected the cells with active caspase-3 at our earliest time point of 6 hours after the onset of ischemia. These cells were scattered throughout the ischemic zone (Fig. 1A). At 24 hours, caspase-3 positive cells significantly increased in numbers with a band of heavy staining through the internal capsule and globus pallidus, with scattered clusters of cells in the cortex close to the surface. Detection of both the newly synthesized procaspase-3 and active caspase- 3 at this time-point, using three different antibodies, revealed the proenzyme synthesis and subsequent activation in the cells primarily in the internal capsule and globus pallidus, with a clearcut physical boundary between caspase-3 positive and negative cells (Fig. 1B, Fig. 2A-B, D-F). Cells with the newly synthesized proenzyme formed the 

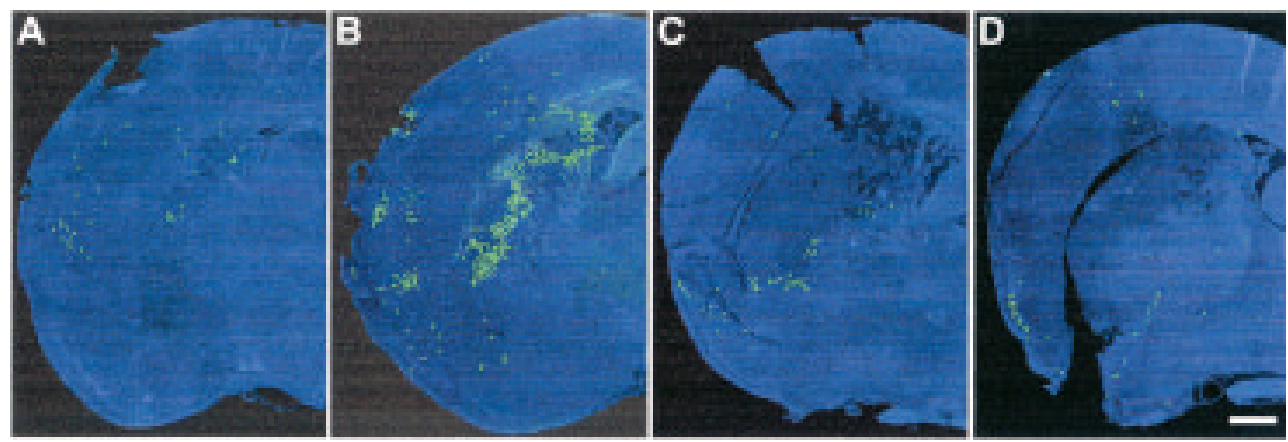

Fig. 1. Active caspase-3 detection (A, B, C, D) in rat brain after focal ischemia (A-6, B-24, C-48, D-72 hours of ischemia). Caspase- 3 detection (green fluorescence) was performed on $6 \mu \mathrm{m}$ thick brain sections. The sections were counterstained with DAPI (blue fluorescence) to visualize cellular DNA. Active caspase-3 had nuclear localization with scattered cells at 6, 48 and 72 h after occlusion. At $24 \mathrm{~h}$, a band of heavy caspase-3 staining occurred through the internal capsule and globus pallidus with scattered cells in the cortex near the surface. Multiple $10 \times$ images were captured using an Olympus IX-70 fluorescent microscope and a MicroMax digital videocamera (Princeton Instruments, Inc. Princeton, NJ, USA). Composite images were created in MetaMorph 4.1 (Advanced Scientific, Inc.). (Bar $=850 \mu \mathrm{m})$

same pattern as the cells with active caspase-3. DFF40/CAD nuclease was also detected in the same areas forming an identical pattern of distribution, with a clear-cut boundary between DFF40/CAD positive and negative cells (Fig. 2C). The clear cut boundary between caspase- 3 positive and negative cells could be an indicator of a steep drop in the oxygen concentration on the edge of the caspase- 3 positive zone, bringing it below the minimal concentration necessary for maintaining caspase- 3 synthesis.

Caspase-3 stained cells did not display welldeveloped morphological signs of apoptosis, probably due to the different dynamics of caspase-3 activation and a later chromatin compaction on the nuclear
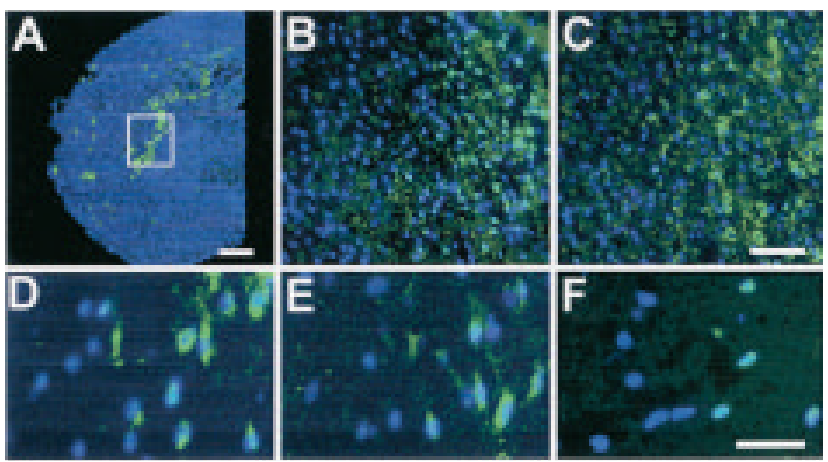

Fig. 2. Active caspase-3 (A, B, F), DFF-40 (C) and procaspase-3 (D, E) synthesis in brain after $24 \mathrm{~h}$ of focal brain ischemia. For caspase- 3 three different antibodies were used, one detecting the pll subunit of procaspase-3 (D), another the p20 subunit (E) and antibody detecting active caspase-3 (A, B, F). Consecutive sections were used and all images (A, B, C, D, E) were taken from the same area (the junction of the internal, facing stroke edge of caspase- 3 positive zone and the external edge of caspase- 3 negative zone inside the frame on $\mathbf{A}$ ). The images demonstrate a distinct boundary between the zone, where procaspase- 3 synthesis and caspase- 3 activation takes place (right side of images) and the more internal areas of stroke (left side of images). The tissue was counterstained with DAPI (blue). (Bar on $\mathbf{A}=1000 \mu \mathrm{m}$; bar on $\mathbf{C}=100 \mu \mathrm{m}$ (refers to $\mathbf{C}$ and $\mathbf{B}$ ); bar on $\mathbf{F}=50 \mu \mathrm{m}$ (refers to $\mathbf{D}, \mathbf{E}, \mathbf{F})$. membrane. However multiple apoptotic bodies were detected in the area of caspase-3 synthesis and activation (Fig. 3 A, D).

After 48 hours of continuing ischemia, caspase3 -positive cells were significantly reduced in numbers (Fig. 1C). At 72 hours after ischemia onset, the numbers of cells with caspase- 3 were reduced even further (Fig. ID). DFF-40 expression followed the same pattern (not shown).

Morphological evaluation of DAPI stained sections taken after $6,24,48$ and 72 hours of permanent ischemia revealed that some cells in the ischemic area displayed clear necrotic morphology (Fig. 4A). Many others, however, displayed extensive apoptotic body formation (Fig. 5). Some cells showed features typical of caspase- 3 independent PCD such as lumpy chromatin condensation on the nuclear membrane $(12,13)$ (Fig. 3A', B', C'). Similar morphology was previously observed in neuronal caspaseindependent apoptosis induced by colchicine treatment (Fig. 3 right panel) (13, with permission from Mol. Med., 2001).

\section{Discussion}

Although extensive and uniform caspase-3 activation throughout the ischemic zone was demonstrated after brain ischemia in neonatal rat (9), an adult rat brain has very low initial levels of procaspase-3, and additional synthesis would be required to achieve the levels similar to those in a neonatal rat brain $(10,11)$. In support of this notion, is the study of Gill et al., which compared procaspase- 3 activity in adult and neonatal models of brain ischemia (14). They demonstrated that there was no activation of procaspase-3 activity in the cytosolic fraction of the brain tissue obtained from the infarcted area of adult rats, whereas, a large increase in this activity was detected in postnatal-day-7 rats (14).

In the present study, we demonstrated positive caspase-3 immunohistochemistry in the ischemic 


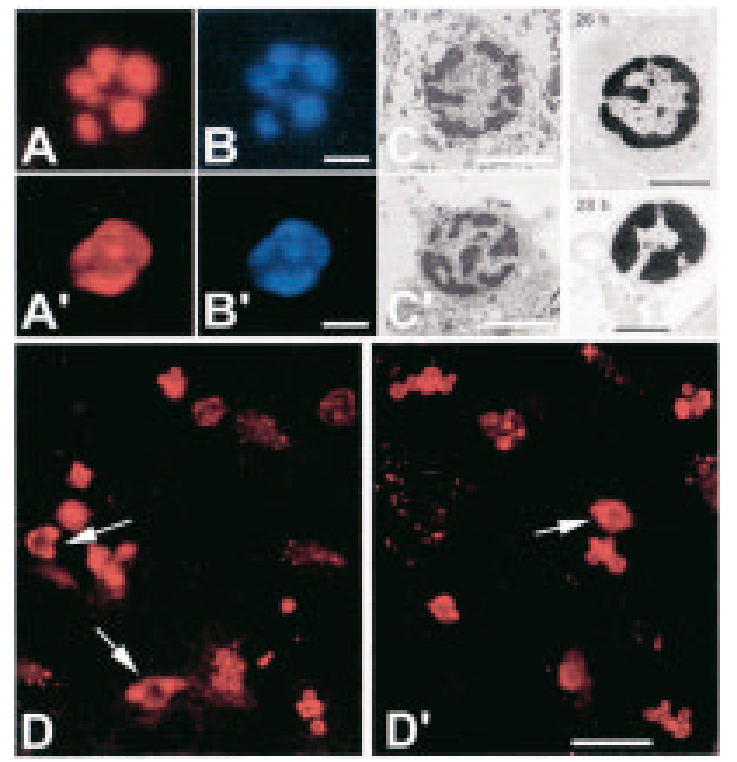

Fig. 3. Fluorescent images of apoptotic cells in the areas of caspase-3 activation $24 \mathrm{~h}$ after onset of ischemia (A, B) and in the areas with caspase-3-independent apoptosis $72 \mathrm{~h}$ after onset of ischemia $\left(\mathbf{A}^{\prime}, \mathbf{B}^{\prime}\right)$. Single color images: $\mathbf{A}, \mathbf{A}^{\prime}-\mathrm{TUNEL}$ (red), B, B'-DAPI (blue). Chromatin condensation, nuclear shrinkage, and apoptotic body formation are present. Fluorescent images were taken at 24 hours $(\mathbf{A}, \mathbf{B})$ and 72 hours $\left(\mathbf{A}^{\prime}, \mathbf{B}^{\prime}\right)$ after the onset of ischemia in caspase- 3 positive and caspase- 3 negative areas respectively. Electron micrographs were obtained from paraffin embedded tissue samples used in histochemical experiments. The sample from the present study was taken from caspase- 3 negative areas (at 6 and $72 \mathrm{hr}$ of ischemia) (C, $\left.\mathbf{C}^{\prime}\right)$, re-embedded in resin and processed for electron microscopic evaluation. The rightmost panel images show nuclei of caspase-inhibited neurons undergoing caspase-independent apoptosis after colchicine treatment (from (13) displaying lumpy, highly condensed chromatin structures. Note the similarity to the morphology of ischemia induced caspase-3 independent apoptosis observed in this study, shown in $\mathbf{C}, \mathbf{C}^{\prime}$. Images $\mathbf{D}$ and $\mathbf{D}^{\prime}$ demonstrate caspase 3 positive (D) and negative $\left(\mathbf{D}^{\prime}\right)$ zones after 24 and 72 hours of ischemia and show bigger TUNEL stained areas. Multiple apoptotic bodies can be seen in both cases. Arrows indicate the cells with chromatin condensation characteristic for apoptosis. (Bar on $\mathbf{B}=5 \mu \mathrm{m}$ (refers to $\mathbf{A}, \mathbf{B}$ ); bar on $\mathbf{B}^{\prime}=5 \mu \mathrm{m}$ (refers to $\mathbf{A}^{\prime}$ and $\mathbf{B}^{\prime}$ ); bar on $\mathbf{C}=5 \mu \mathrm{m}$; bar on $\mathbf{C}^{\prime}=5 \mu \mathrm{m}$; bars on the rightmost panel images from (13) $=2 \mu \mathrm{m}$, bar on $\mathbf{D}^{\prime}=25 \mu \mathrm{m}$ (includes $\mathbf{D}$ and $\mathbf{D}^{\prime}$ )

adult rat brain beginning at 6 hours post occlusion, peaking at 24 hours post occlusion, and still somewhat visible at 72 hours post occlusion. The results of this study are generally consistent with other immunohistochemical reports of caspase 3 expression following permanent focal cerebral ischemia in adult rats, although some temporal and anatomical differences exist (15-17). Velier et al. examined active caspase 3 staining between 6 hours and 5 days post occlusion (15). They found some positive staining at 6 hours, and by 24 hours they found clusters of neuronal staining in the cortex. They did not, however, report whether subcortical staining was apparent, as was found in the present study. Krupinski and colleagues sacrificed rats as early as 30 minutes and as late as 4 days post occlusion (16). They found visible procaspase 3 staining at 30 minutes that was strongest at 12-24 hours, but was still visible at 48 hours. Positive staining was seen in the cortex as well as subcortical areas such as the corpus collosum, striate, thalamus and hippocampus. Sasaki et al measured caspase-3 staining in the core and penumbra of the infarct (17). They report that caspase-3 staining was strongest in the penumbra at 8 hours post occlusion and was beginning to decline by $24 \mathrm{~h}$ after occlusion. These differences in caspase- 3 expression are minimal and could well be due to differences in techniques of stroke induction.

In the current study, we demonstrated the pattern of caspase staining in the entire ischemic hemisphere, providing a large-scale perspective unavailable in the previous reports. Such reconstruction permits the assessment of the patterns of caspase-3 activation throughout the brain.

Our data further indicate that, while a number of cells in the ischemic zone upregulate their procaspase- 3 and caspase- 3 levels with subsequent activation of DFF40/CAD nuclease following permanent focal brain ischemia in adult rats, many others do not. A significant number of cells in the ischemic zone do not express caspase-3, yet only some of them are necrotic, while others display non-classical apoptotic morphology with apoptotic body formation. The presence of this morphology is not accompanied by either synthesis or activation of caspase- 3 and DFF-40 activation.

Similar to our data, a delay in the onset, but not a decrease, in apoptosis was observed in NT2 human neuronal cells after caspase-3 inhibition (18) and in cultured neurons from mice lacking caspase-3 (19).

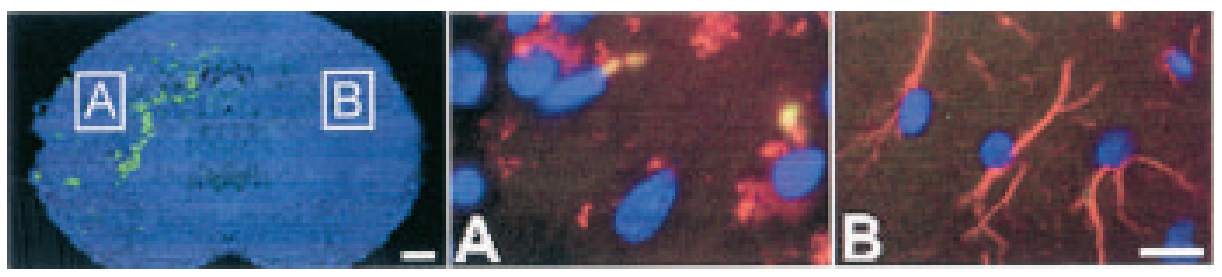

Fig. 4. Necrotic cells in caspase-3 negative zone after $24 \mathrm{~h}$ ischemia. (A) Necrotic neurons are identified by the presence of neuronal cell marker tubulin (Red). Swollen nuclei are visualized by DAPI staining (Blue). Normal cells in the contralateral hemisphere are shown for comparison (B). The low magnification image of the brain on the left shows the areas where images $\mathbf{A}$ and $\mathbf{B}$ were taken from. (Bar on the low magnification brain image $=1000 \mu \mathrm{m}$; bar on $\mathbf{B}=20 \mu \mathrm{m}$ (refers to $\mathbf{A}, \mathbf{B}$ ). 


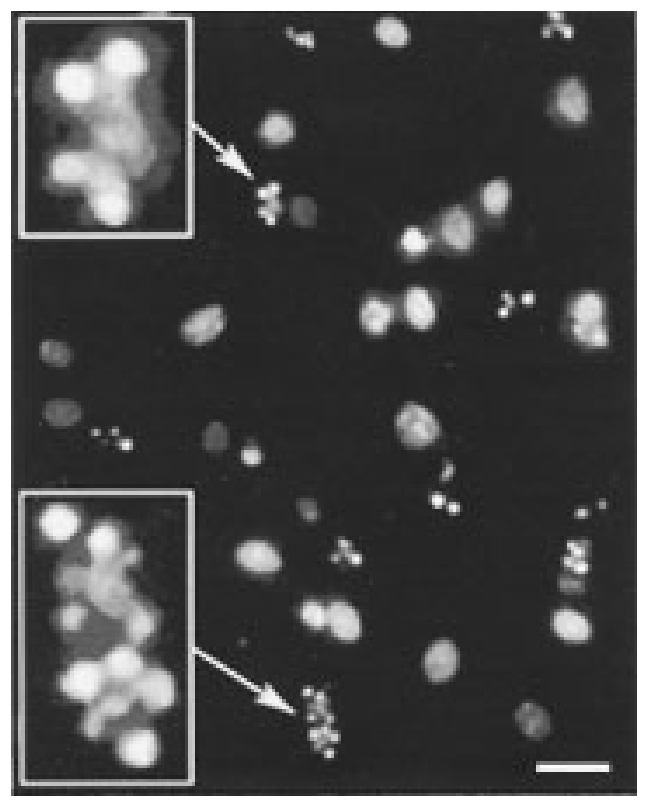

Fig. 5. Apoptotic cells with multiple apoptotic bodies in a region with no caspase-3 staining at $24 \mathrm{~h}$ of ischemia. The sections were stained by DAPI to visualize cellular DNA. $($ Bar $=25 \mu \mathrm{m})$.

Additionally, in normal mouse neurons driven to apoptosis, the partial protective effect of caspase-3 inhibition is not seen beyond $24 \mathrm{hr}$, suggesting that caspase-3 inhibition delays but does not prevent apoptotic cell death (19).

It is possible that the role caspase- 3 plays in apoptosis of brain cells depends on the physiological situation. A specific feature of adult rat brain is that under normal conditions, its cells maintain very low levels of procaspase-3 $(10,11)$. In order to execute an apoptotic program and to disassemble itself, the brain cell needs to perform caspase-3 synthesis requiring energy expenditures. In this regard, ischemia induced apoptosis faces unusual demands, as it occurs in cells often depleted of energy. Though a short period of ischemia is sufficient to prime cells to undergo programmed cell death (20), there is a substantial difference between the apoptotic process only primed by an ischemic episode and occurring in conditions of restored oxygen supply, and apoptosis developing under conditions of persistent energy depletion.

Nevertheless, caspase- 3 is highly inducible by various factors, including ischemia. Permanent middle cerebral artery occlusion in rat results in a rapid activation of procaspase mRNA expression, with a $41 \%$ increase at 6 hours and a $220 \%$ increase by 24 hours (21). However, as we have shown, this activation does not occur in all cells that appear to undergo apoptosis.

It is now apparent that cell death can take many paths following cerebrovascular occlusion, and that these varied paths result in variations in neuronal morphology $(22,23)$. Following occlusion, some cells may undergo classical PCD, with caspase-3 activation and subsequent cytoplasmic and nuclear condensations and compartmentalization. However, various death routines can be activated following occlusion, resulting in neurons with mixed apoptotic and necrotic morphology $(12,22,23)$.

These variations have prompted some investigators to adopt a more extensive characterization of cell death morphology, which includes different patterns of death $(12,22-24)$. In classical apoptosis, stereotypic apoptotic morphology is present, and includes nuclear chromatin compartmentalization into simple geometric shapes. Caspase activation and the well-defined molecular apoptotic pathways appear critical for this morphology to develop $(12,13)$. In apoptosis-like programmed cell death, death signaling pathways result in chromatin compaction into irregular, lumpy geometric shapes. This nuclear morphology can occur in the presence of caspase activation but is commonly seen in caspase-independent apoptosis, and is often mediated by other proteases (13). This is the apoptotic morphology seen in the caspase-negative areas in the present study. Necrosis-like programmed cell death generally occurs in the absence of caspase activation and occurs without chromatin compaction. Execution of the necrotic cell death program may be due to PARP or Fas signaling $(25,26)$. Accidental necrosis is purely stimulusdriven and is characterized by cellular edema and organelle swelling (12).

Our data indicates that in conditions of persisting ischemia, many cells display some morphological markers of apoptosis and self-dismantle in apoptotic-like fashion with chromatin condensation and apoptotic body formation, but without caspase-3 synthesis and activation. However, in other cells, caspase- 3 synthesis and activation do occur.

The fact that ischemic brain cells, having no active caspase-3, can still proceed to apoptotic death contributes to an understanding of the limited efficacy of anti-ischemic drugs targeting caspase-3, and is an important phenomenon to consider when developing therapeutic strategies to control ischemiainduced apoptosis. The results of this study suggest that the suppression of caspase- 3 synthesis and activation in treatment of ischemic brain injury may meet with only modest success, because an alternative pathway or pathways to apoptotic cell death can be activated in focal brain ischemia.

\section{Acknowledgments}

This research was supported by grant R01 CA7891201 from the National Cancer Institute, National Institutes of Health (DSB), by grant 004949-054 from the Texas Higher Education Coordinating Board (DSB), by a grant from the Veterans Affairs Merit Review Board (DSB), and by grants from DeBakey Medical Foundation (VVD), and Baylor College of Medicine (VVD). Additional support (DSB) was 
provided from The Taub Foundation, The Henry J.N. Taub Fund for Neurosurgical Research, The George A. Robinson, IV Foundation, The Blanche Greene Estate Fund of The Pauline Sterne Wolff Memorial Foundation, and The Seigo Arai and Koppelman Funds of The Neurological Research Foundation.

\section{References}

1. Porter AG, Janicke RU. (1999) Emerging roles of caspase-3 in apoptosis. Cell Death Differ. 6: 99-104.

2. Mukae N, Enari M, Sakahira H, et al. (1998) Molecular cloning and characterization of human caspase-activated DNase. Proc. Natl. Acad. Sci. USA 95: 9123-9128.

3. Liu X, Zou H, Slaughter C, Wang X. (1997) DFF, a heterodimeric protein that functions downstream of caspase- 3 to trigger DNA fragmentation during apoptosis. Cell 89: 175-184.

4. Enari M, Sakahira H, Yokoyama H, et al. (1998) A caspaseactivated DNase that degrades DNA during apoptosis, and its inhibitor ICAD. Nature 391: 43-50.

5. Sakahira H, Enari M, Nagata S. (1998) Cleavage of CAD inhibitor in CAD activation and DNA degradation during apoptosis. Nature 391: 96-99.

6. Eldadah BA, Ren RF, Faden AI. (2000) Ribozyme-mediated inhibition of caspase-3 protects cerebellar granule cells from apoptosis induced by serum-potassium deprivation. J. Neurosci. 20: 179-186.

7. Wiessner C, Sauer D, Alaimo D, Allegrini PR. (2000) Protective effect of a caspase inhibitor in models for cerebral ischemia in vitro and in vivo. Cell. Mol. Biol. 46: 53-62.

8. Hara H. (1999) Involvement of caspase on apoptosis in ischemia-induced neuronal cell death: usefulness of caspase inhibitors for stroke therapy. Nippon Yakurigaku Zasshi 113: 97-1 11.

9. Han BH, D'Costa A, Back SA, et al. (2000) BDNF blocks caspase-3 activation in neonatal hypoxia-ischemia. Neurobiol. Dis. 7: 38-53.

10. Ni B, Wu X, Su Y, et al. (1998) Transient global forebrain ischemia induces a prolonged expression of the caspase- 3 mRNA in rat hippocampal CAl pyramidal neurons. J. Cereb. Blood Flow Metab. 18: 248-256.

11. Shimohama S, Tanino H, Fujimoto S. (1999) Changes in caspase expression in Alzheimer's disease: comparison with development and aging. Biochem. Biophys. Res. Commun. 256: 381-384.

12. Leist M, Jäättelä M. (2001) Four deaths and a funeral: from caspases to alternative mechanisms. Nature Reviews 2: 1-10.
13. Volbracht C, Leist M, Kolb SA, Nicotera P. (2001) Apoptosis in caspase-inhibited neurons. Mol. Med. 7(1): 36-48.

14. Gill R, Soriano M, Blomgren K, et al. (2002) Role of caspase3 activation in cerebral ischemia-induced neurodegeneration in adult and neonatal brain. J. Cereb. Blood Flow Metab. 22: 420-430.

15. Velier JJ, Ellison JA, Kikly KK, et al. (1999) Caspase-8 and Caspase-3 Are Expressed by Different Populations of Cortical Neurons Undergoing Delayed Cell Death after Focal Stroke in the Rat. J. Neurosci, 19: 5932-5941.

16. Krupinski J, Lopez E, Marti E, Ferrer I. (2000) Expression of caspases and their substrates in the rat model of focal cerebral ischemia. Neurobiology of Disease 7: 332-342.

17. Sasaki C, Kitagawa H, Zhang WR, et al. (2000) Temporal profile of cytochrome $\mathrm{c}$ and caspase- 3 immunoreactivities and TUNEL staining after permanent middle cerebral artery occlusion in rats. Neurological Research 22: 223-228.

18. Walker PR, Leblanc J, Carson C, et al. (1999) Neither caspase-3 nor DNA fragmentation factor is required for high molecular weight DNA degradation in apoptosis. Ann. N.Y. Acad. Sci. 887: $48-59$.

19. D'Mello SR, Kuan CY, Flavell RA, Rakic PJ. (2000) Caspase-3 is required for apoptosis-associated DNA fragmentation but not for cell death in neurons deprived of potassium. $\mathrm{J}$. Neurosci. Res. 59: 24-31.

20. Endres M, Namura S, Shimizu-Sasamata M, et al. (1998) Attenuation of delayed neuronal death after mild focal ischemia in mice by inhibition of the caspase family. J. Cereb. Blood Flow Metab. 18: 238-247.

21. Harrison DC, Medhurst AD, Bond BC, et al. (2000) The use of quantitative RT-PCR to measure mRNA expression in a rat model of focal ischemia-caspase- 3 as a case study. Brain Res. Mol. Brain Res. 75: 143-149.

22. Benchoua A, Guegan C, Couriaud C, et al. (2001) Specific caspase pathways are activated in the two stages of cerebral infarction. J. Neurosci. 21: 7127-7134.

23. Roy M, Sapolsky R. (1999) Neuronal apoptosis in acute necrotic insults: why is this subject such a mess? TINS 22: 419-422.

24. Niciotera P, Leist M, Manzo L. (1999) Neuronal cell death: a demise with different shapes. TIPS 20: 46-51.

25. Holler N, et al. (2000) Fas triggers an alternative, caspase8 -independent cell death pathway using the kinase RIP as effector molecule. Nature Immunol. 1: 489-495.

26. Ha HC, Snyder SH. (1999) Poly(ADP-ribosome) polymerase is a mediator of necrotic-cell death by ATP depletion. Proc. Nat. Acad. Sci. USA 96: 13978-13982. 